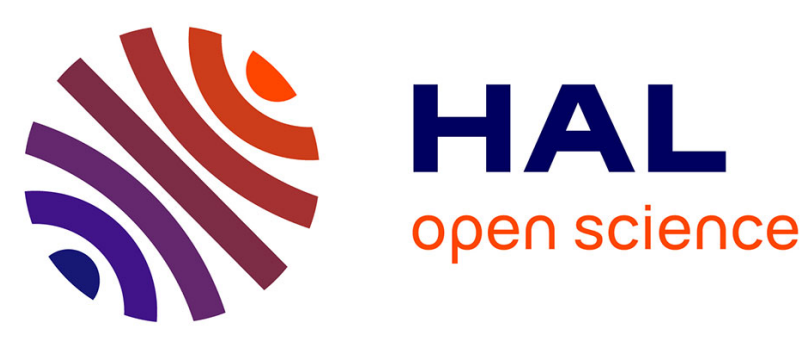

\title{
The SPOTIS Rank Reversal Free Method for Multi-Criteria Decision-Making Support
}

Jean Dezert, Albena Tchamova, Deqiang Han, Jean-Marc Tacnet

\section{To cite this version:}

Jean Dezert, Albena Tchamova, Deqiang Han, Jean-Marc Tacnet. The SPOTIS Rank Reversal Free Method for Multi-Criteria Decision-Making Support. 2020 IEEE 23rd International Conference on Information Fusion (FUSION), Jul 2020, Sun City, South Africa. pp.1-8, 10.23919/FUSION45008.2020.9190347 . hal-03007675

\section{HAL Id: hal-03007675 https://hal.science/hal-03007675}

Submitted on 16 Nov 2020

HAL is a multi-disciplinary open access archive for the deposit and dissemination of scientific research documents, whether they are published or not. The documents may come from teaching and research institutions in France or abroad, or from public or private research centers.
L'archive ouverte pluridisciplinaire HAL, est destinée au dépôt et à la diffusion de documents scientifiques de niveau recherche, publiés ou non, émanant des établissements d'enseignement et de recherche français ou étrangers, des laboratoires publics ou privés. 


\section{The SPOTIS Rank Reversal Free Method for Multi-Criteria Decision-Making Support}

\author{
Jean Dezert \\ The French Aerospace Lab \\ Palaiseau, France. \\ jean.dezert@onera.fr
}

\author{
Albena Tchamova \\ Inst. of I\&C Tech., BAS \\ Sofia, Bulgaria. \\ tchamova@bas.bg
}

\author{
Deqiang Han \\ Inst. of Integrated Automation \\ Xi' an Jiaotong Univ., China. \\ deqhan@xjtu.edu.cn
}

\author{
Jean-Marc Tacnet \\ INRAE, U.R. ETNA \\ 38000 Grenoble, France. \\ jean-marc.tacnet@inrae.fr
}

\begin{abstract}
In this paper, we propose a new Multi-Criteria Decision-Making method (MCDM) which is rank reversal free. We call it the SPOTIS method standing for Stable Preference Ordering Towards Ideal Solution method. Our method is exempt of rank reversal because the preference ordering established from the score matrix of the MCDM problem under consideration does not require the relative comparisons between alternatives, but only comparisons with respect to the ideal solution chosen by the MCDM system designer after transforming the incomplete original MCDM problem into a well-defined one thanks to the specification of the min and max bounds of each criterion involved in the problem.
\end{abstract}

Keywords: SPOTIS, multi-criteria decision-making, Information fusion, TOPSIS, MCDM.

\section{INTRODUCTION}

Multi-Criteria Decision-Making (MCDM) is to choose an alternative among a given set of alternatives based on their quantitative evaluations (numerical scores) obtained with respect to different criteria. Because the scores are usually expressed in different (physical) units and scales, a normalization step is often used in the development of MCDM methods available in the literature, which is recognized as a source of the so-called Rank Reversal Problem (RRP) [1]-[10]. Rank reversal is a change of the rank (or preference ordering) of alternatives if we change the structure of the MCDM problem by adding (or deleting) some alternative. The recent survey paper [11] provides a very good detailed literature review of RRP in MCDM. The most adopted MCDM methods used so far are $\mathrm{AHP}^{1}$ [12], ELECTRE ${ }^{2}$ [7], TOPSIS ${ }^{3}$ [13], [14] which all suffer from rank reversal. Other MCDM methods have been inspired by these methods trying to overcome more or less successfully RRP. In 2013, a new MCDM method avoiding data normalization called Estimator Ranking Vector (ERV) has been presented in [15] which unfortunately is not exempt of RRP. Quite recently an interesting Rank Reversal Free (RRF) method, called COMET (Characteristic Object METhod) has been proposed by Piegat and Sałabun [16]-[18] to address MCDM from a fuzzy logic standpoint. COMET method avoids rank reversal, which is very appealing, but it requires much

\footnotetext{
${ }^{1}$ Analytic Hierarchy Process.

${ }^{2}$ ELimination and/Et Choice Translating REality.

${ }^{3}$ Technique for Order Preference by Similarity to Ideal Solution.
}

more information ${ }^{4}$ than the given score matrix used classically in MCDM problems. So, in our opinion, COMET approach must not be compared with classical methods because it requires more information than we usually have in classical MCDM problems.

In this paper, we propose a new rank reversal free MCDM method which provides a Stable Preference Ordering Towards Ideal Solution (SPOTIS) and has a very low complexity. It requires much less information with respect to the COMET approach. Moreover SPOTIS fits easily in the framework of classical MCDM problematic because it uses directly the MCDM score matrix available, and the importance weighting factors of criteria.

The paper is organized as follows. After a brief recall of basics of the classical MCDM problem in section II, we present the principle of the new SPOTIS method in section III. The application of SPOTIS method to a multi-criteria car selection problem is presented in section IV with comparison to AHP, TOPSIS and Belief Function based TOPSIS methods. In section $\mathrm{V}$ we present briefly the SPOTIS method for working with an expected solution point. Conclusions, and perspectives are given in the section VI.

\section{BASICS ON CLASSICAL MCDM}

A Multi-Criteria Decision-Making problem is characterized by a set of alternatives $\mathbf{A} \triangleq\left\{A_{1}, A_{2}, \ldots, A_{M}\right\}(M>2)$ in which the best decision must be made, according to a given set of criteria $\mathbf{C} \triangleq\left\{C_{1}, C_{2}, \ldots, C_{N}\right\}(N \geq 1)$ and the score $M \times N$ matrix $\mathbf{S}=\left[S_{i j}\right]$ whose component $S_{i j}$ is the score (the performance) of the alternative $A_{i}$ based on criterion $C_{j}$. Each criterion has an importance normalized weight $w_{j} \in[0,1]$ with $\sum_{j=1}^{N} w_{j}=1$. The MCDM problem is said to be classical if all criteria $C_{j}$ and all alternatives $A_{i}$ are known as well as all their related scores values $S_{i j}$ expressed quantitatively (i.e. $S_{i j}$ are real numbers) and the weighting factor $w_{j}$ of each criteria $C_{j}$. Unclassical MCDM problems refer to problems involving incomplete or qualitative information. The set of normalized weighting factors is denoted by $\mathbf{w} \triangleq\left\{w_{1}, w_{2}, \ldots, w_{N}\right\}$. Depending on the context of the MCDM problem, the score can be interpreted either as a cost (or expense) or as a reward

\footnotetext{
${ }^{4}$ More precisely, the a priori choice of fuzzy set membership functions, and sets of particular characteristics values for each criterion.
} 
(or benefit). Further on, by convention and without the loss of generality ${ }^{5}$ we will interpret the score as a reward having monotonically increasing preference. Thus, the best alternative w.r.t. ${ }^{6}$ a given criterion will be the one providing the highest reward/benefit. The score matrix $\mathbf{S}=\left[S_{i j}\right]$ is sometimes also called benefit or payoff matrix in the literature. The classical MCDM problem aims to select the best ${ }^{7}$ alternative (corresponding to the most preferred one) $A^{\star} \in \mathbf{A}$ given $\mathbf{S}$ and the weighting factors $\mathbf{w}$ of criteria.

It is worth noting that the classical MCDM problem based only on the knowledge of a given score matrix $\mathbf{S}$ and an importance weighting vector $\mathbf{w}$ of criteria is in fact an incomplete MCDM problem because the absolute (or physical) bounds of the score values of each criterion are not specified, and we consider that most of MCDM problems are actually illdefined (i.e. incompletely specified) problems. In [19] (p. 148 $\&$ p.175), the author adopts a more radical standpoint and stresses the fact that MCDM problems are, and will always be ill-defined problems because it is difficult, or impossible, to gather all relevant technical parameters, and to validate them against the observations.

To fully characterize and solve a MCDM problem, one also needs to know (or to specify) the absolute bounds of the score values of each criterion so that the (ill-defined) classical MCDM problem becomes a well-defined MCDM one, where all scores values for each criterion are between its bounds. Later in the paper we propose a direct and very simple method to solve well-defined MCDM problems thanks to SPOTIS method. Of course transforming an ill-defined MCDM problem into a well-defined MCDM one requires extra information which is sometimes already available but not exploited in well-known methods, or which should be introduced based on reasonable assumptions or expert elicitation depending on the criteria involved in the ill-defined MCDM problem. Once the ill-defined MCDM problem is transformed into a unique well-defined one, the rank reversal free SPOTIS method will provide the best multi-criteria decision-making solution with preference ordering of all alternatives.

\section{SPOTIS METHOD}

In this paper, we always consider the criteria independent from each other so that no (even partially) redundant information is used in the MCDM problem to avoid some bias of the result. The principle SPOTIS method is based on the computation of normalized distance $d_{i j}\left(A_{i}, S_{j}^{\star}\right)$ of each alternative $A_{i}$ with respect to the (best) ideal solution $S_{j}^{\star}$ chosen for each criterion $C_{j}$, and their weighted average distance $d\left(A_{i}, S^{\star}\right)=\sum_{j=1}^{N} w_{j} d_{i j}\left(A_{i}, S_{j}^{\star}\right)$ which is also a true distance metric because of the following Theorem.

Theorem 1: Consider $N \geq 2$ metric spaces $E_{1}, E_{2}, \ldots, E_{N}$. We denote $d_{j}\left(x_{j}, y_{j}\right)$ as a true metric chosen for measuring

\footnotetext{
${ }^{5}$ Indeed the cost score value can be interpreted as benefit by multiplying its value by -1 .

${ }^{6}$ with respect to.

${ }^{7}$ In some sense that will be shortly clarified later in the paper.
}

the distance between points $x_{j}$ and $y_{j}$ of $E_{j}$. We consider $N$-dimensional points defined as $\mathbf{x}=\left[\begin{array}{llll}x_{1} & x_{2} & \ldots & x_{N}\end{array}\right]^{t}$ and $\mathbf{y}=\left[\begin{array}{llll}y_{1} & y_{2} & \ldots & y_{N}\end{array}\right]^{t}$ belonging to $E=E_{1} \times E_{2} \times \ldots \times E_{N}$. Then for any real factor $w_{j} \geq 0$, the weighted average distance $d(\mathbf{x}, \mathbf{y})$ defined by $d(\mathbf{x}, \mathbf{y})=\sum_{j=1}^{N} w_{j} d_{j}\left(x_{j}, y_{j}\right)$ is a true distance.

\section{Proof: See Appendix.}

To avoid rank reversal, the ideal solution $S^{\star}$ must be chosen a priori and independently of score values of alternatives so that the distance $d\left(A_{i}, S^{\star}\right)$ of any chosen alternative $A_{i}$ to $S^{\star}$ is independent of the distance of $d\left(A_{i^{\prime}}, S^{\star}\right)$ for $i^{\prime} \neq i$ as already pointed out by Kong [20]. By doing this, the preference ordering based on $d\left(A_{i}, S^{\star}\right)$ will be stable (i.e. exempt of rank reversal) because adding or removing some alternatives of the set $\mathbf{A}$ of a given MCDM problem will not change the values $d\left(A_{i}, S^{\star}\right)$ of the modified MCDM problem, as it will be shown in our examples.

\section{A. Choice of an ideal solution point}

Usually the (best) Ideal Solution Point (ISP) is determined from the bounds of the scores values of the criteria according to the preference ordering related to each criterion. However in some MCDM problems the ideal solution point can also be chosen as some "expected" (or nominal) reference point between these bounds. In fact, the choice of the ISP is left to the MCDM system designer and the type of MCDM problems he/she wants to address. The MCDM problem consists in choosing (or sorting) the alternatives with respect to the defined ideal solution point. The closer to ISP, the better the MCDM solution.

For each criteria $C_{j}(j=1,2, \ldots, N)$ the $\min$ and $\max$ bounds of this criterion are denoted respectively by $S_{j}^{\text {min }}$ and $S_{j}^{\max }$. If for a criterion $C_{j}$ the preference is larger score value is better, then the best ideal solution for criterion $C_{j}$ is $S_{j}^{\star}=$ $S_{j}^{\max }$, but if for criterion $C_{j}$ the preference is smaller score value is better, then the ideal solution point for criterion $C_{j}$ is $S_{j}^{\star}=S_{j}^{\text {min }}$. The ideal multi-criteria best solution $S^{\star}$ is defined as the point of coordinates $\left(S_{1}^{\star}, \ldots, S_{j}^{\star}, \ldots, S_{N}^{\star}\right)$ in the $\mathrm{N}$-dimensional space.

Example 1: Consider a simple classical MCDM problem with 4 alternatives and 3 criteria with weighting vector $\mathbf{w}=$ $\left[\begin{array}{lll}0.2 & 0.3 & 0.5\end{array}\right]$ and with the score matrix given by

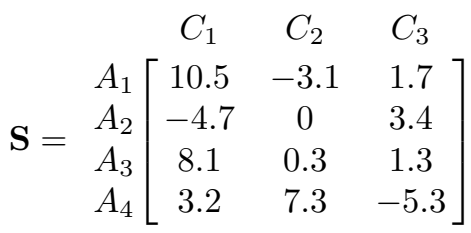

This classical MCDM problem is clearly incomplete because the bounds of the values of the scores are not specified. It becomes a well-defined MCDM problem if one specifies the min and max bounds of score values for each criterion. For instance, one could consider as reasonable (of course this 
choice depends on the type of application and criteria under concern) the following bounds

$$
\begin{aligned}
& {\left[S_{1}^{\min }, S_{1}^{\max }\right]=[-5,12]} \\
& {\left[S_{2}^{\min }, S_{2}^{\max }\right]=[-6,10]} \\
& {\left[S_{3}^{\min }, S_{3}^{\max }\right]=[-8,5]}
\end{aligned}
$$

If for criteria $C_{1}$ and $C_{3}$ the preference is larger score value is better, and for criterion $C_{2}$ the preference is smaller score value is better then the ideal best solution is given by

$$
S^{\star}=\left(S_{1}^{\star}, S_{2}^{\star}, S_{3}^{\star}\right)=\left(S_{1}^{\max }, S_{2}^{\min }, S_{3}^{\max }\right)=(12,-6,5)
$$

Of course any other ISP can be chosen depending on the MCDM problem one wants to solve. For example, if we are rather interested by finding the alternative which is the closest to the mid score value of each criterion then we will use the expected solution point $S^{\star}=(8.5,8,6.5)$ because 8.5 is the middle of $\left[S_{1}^{\min }, S_{1}^{\max }\right]=[-5,12], 8$ is the middle of $\left[S_{2}^{\min }, S_{2}^{\max }\right]=[-6,10]$, and 6.5 is the middle of $\left[S_{3}^{\min }, S_{3}^{\max }\right]=[-8,5]$.

\section{B. Choice of distance metric}

To measure the closeness of an alternative $A_{i}(i=$ $1,2, \ldots, M)$ with respect to the ideal point solution, we will use the weighted average distance $d\left(A_{i}, S^{\star}\right)=$ $\sum_{j=1}^{N} w_{j} d_{i j}\left(A_{i}, S_{j}^{\star}\right)$ which is proved to be a true distance metric thanks to Theorem 1 . This theorem is very general and it does not require that all distances $d_{i j}\left(A_{i}, S_{j}^{\star}\right)$ for $j=1,2, \ldots, N$ involved in the weighted average must be of the same kind. For instance, one may chose a city-block $\left(L_{1}\right)$ distance for measuring the distance in $E_{1}$ metric space, and one may chose an Euclidean $\left(L_{2}\right)$ distance for measuring the distance in $E_{2}$ metric space, and another possible Minkowski's distance related with $E_{3}$, etc. Although it is mathematically allowed to work with such type of mixed/hybrid weighted average distance, we do not see a very solid justification ${ }^{8}$ for doing this a priori, and that is why we propose to use the same distance metric for each criterion. Further on we will use the classical Euclidean $\left(L_{2}\right)$ distance [21] for calculating $d_{i j}\left(A_{i}, S_{j}^{\star}\right)$, but any other choice of distances is possible, and is theoretically allowed in SPOTIS method (including the hybrid weighted averaged distance).

\section{On the necessity of normalization}

Another question concerns the necessity, or not, to normalize the score values (or eventually the distances values) relatively to each criterion before computing the weighted average distance $d\left(A_{i}, S^{\star}\right)=\sum_{j=1}^{N} w_{j} d_{i j}\left(A_{i}, S_{j}^{\star}\right)$ that will help to sort the alternatives with respect to the ideal solution point. Although not absolutely necessary from the mathematical standpoint, it seems natural and preferable to apply a normalization step before computing the weighted average distance $d\left(A_{i}, S^{\star}\right)$ mainly because the criteria have usually

\footnotetext{
${ }^{8}$ Maybe for particular types of criterion some distance metrics are more appropriate than other but we did not investigate this question yet.
}

very different natures ${ }^{9}$ characterized by different (physical) units. In fact, it seems very difficult to define a clear semantics (if any) for a weighted average distance that mixes distances of objects of different natures. To circumvent this problem, we prefer to apply a normalization step to work with unitless $d_{i j}\left(A_{i}, S_{j}^{\star}\right)$ distances involved in weighted average distance $d\left(A_{i}, S^{\star}\right)=\sum_{j=1}^{N} w_{j} d_{i j}\left(A_{i}, S_{j}^{\star}\right)$.

Let's examine two natural and simple normalization procedures, and their relationships.

- First possible normalization procedure

The first normalization to make the score values related to a criteria $C_{j}$ unitless consists in normalizing the score value $S_{i j}$ by taking

$$
s_{i j}=\frac{S_{i j}-S_{j}^{\min }}{S_{j}^{\max }-S_{j}^{\min }}
$$

Hence the normalized score value $s_{i j} \in[0,1]$, and $s_{i j}=0$ if $S_{i j}=S_{j}^{\mathrm{min}}$, and $s_{i j}=1$ if $S_{i j}=S_{j}^{\max }$. Of course the coordinates of the ideal solution point must be also normalized to get the normalized ISP $s^{\star}=\left(s_{1}^{\star}, \ldots, s_{j}^{\star}, \ldots, s_{N}^{\star}\right)$, where $s_{j}^{\star}=\frac{S_{j}^{\star}-S_{j}^{\min }}{S_{i}^{\max }-S_{\dot{m}}^{\min }}$ for $j=1,2, \ldots, N$. Hence the original Euclidean distance $d_{i j}\left(A_{i}, S_{j}^{\star}\right)$ defined by ${ }^{10}$

$$
d_{i j}\left(A_{i}, S_{j}^{\star}\right)=\left|S_{i j}-S_{j}^{\star}\right|
$$

will be replaced by the unitless normalized Euclidean distance $d_{i j}\left(A_{i}, s_{j}^{\star}\right) \in[0,1]$ defined by

$$
d_{i j}\left(A_{i}, s_{j}^{\star}\right)=\left|s_{i j}-s_{j}^{\star}\right|
$$

It is worth noting that one has also ${ }^{11}$

$$
\begin{aligned}
d_{i j}\left(A_{i}, s_{j}^{\star}\right) & =\left|\frac{S_{i j}-S_{j}^{\min }}{S_{j}^{\max }-S_{j}^{\min }}-\frac{S_{j}^{\star}-S_{j}^{\min }}{S_{j}^{\max }-S_{j}^{\min }}\right| \\
& =\frac{\left|S_{i j}-S_{j}^{\star}\right|}{\left|S_{j}^{\max }-S_{j}^{\min }\right|}
\end{aligned}
$$

Once the normalized distances $d_{i j}\left(A_{i}, s_{j}^{\star}\right)$ are calculated, we compute the normalized weighted average distance $d\left(A_{i}, s^{\star}\right) \in[0,1]$ defined by

$$
d\left(A_{i}, s^{\star}\right)=\sum_{j=1}^{N} w_{j} d_{i j}\left(A_{i}, s_{j}^{\star}\right)
$$

to sort alternatives with respect to the (normalized) ISP.

- Second possible normalization procedure

In this second possible normalization, we do not normalize the scores values directly but only the calculated distances with respect to min and max distances values.

\footnotetext{
${ }^{9}$ For instance a criterion can refer to time, another criterion can refer to price, another one to dimension, etc.

${ }^{10}$ In our context we work in one dimension for each criterion, so that the Euclidean distance $d(x, y)=\sqrt{(x-y)^{2}}=|x-y|$.

${ }^{11}$ Just replace expressions for $s_{i j}$ and $s_{j}^{\star}$ in (2).
} 
That is, we compute for each alternative $A_{i}$ and criterion $C_{j}$ at first the Euclidean distance defined by

$$
d_{i j}\left(A_{i}, S_{j}^{\star}\right)=\left|S_{i j}-S_{j}^{\star}\right|
$$

and then we normalize its value by taking

$$
\tilde{d}_{i j}\left(A_{i}, S_{j}^{\star}\right)=\frac{d_{i j}\left(A_{i}, S_{j}^{\star}\right)-d_{j}^{\min }}{d_{j}^{\max }-d_{j}^{\min }}
$$

where $d_{j}^{\text {min }}$ corresponds to the minimum achievable distance which is zero only if the score value $S_{i j}$ coincides with the ideal solution $S_{j}^{\star}$. The maximum distance is $d_{j}^{\max }=\left|S_{j}^{\max }-S_{j}^{\min }\right|$ because $S_{i j} \in\left[S_{j}^{\min }, S_{j}^{\max }\right]$. Hence, the formula (5) can be written as

$$
\tilde{d}_{i j}\left(A_{i}, S_{j}^{\star}\right)=\frac{d_{i j}\left(A_{i}, S_{j}^{\star}\right)}{\left|S_{j}^{\max }-S_{j}^{\min }\right|}=\frac{\left|S_{i j}-S_{j}^{\star}\right|}{\left|S_{j}^{\max }-S_{j}^{\min }\right|}
$$

Once the normalized distances are calculated, we compute the normalized weighted average distance $\tilde{d}\left(A_{i}, S^{\star}\right) \in[0,1]$ defined by

$$
\tilde{d}\left(A_{i}, S^{\star}\right)=\sum_{j=1}^{N} w_{j} \tilde{d}_{i j}\left(A_{i}, S_{j}^{\star}\right)
$$

to sort the alternatives with respect to the ISP.

One can easily verify that the two normalization procedures are in fact equivalent because the formulas (3) and (6) are the same. Hence, we can either use the normalization procedure 1 or 2 as one prefers. It does not matter because one will always have $d\left(A_{i}, s^{\star}\right)=\tilde{d}\left(A_{i}, S^{\star}\right)$. We recall that the weighting factor $w_{j}$ entering in (4) and in (7) is the importance weighting factor of the $j$-th criterion which is chosen independently of the score values and the bounds of the criterion.

\section{Choice of the bounds}

SPOTIS method needs extra information on the bounds of criteria in order to transform the original ill-defined MCDM problem into a well-defined MCDM problem to obtain its solution. We do not know yet if there exists, or not, a general principle for automatic bound selection for the SPOTIS method, and this is a challenging open question. A priori it appears difficult to establish very general principles because the bound selection appears very dependent of the nature of criteria involved in the MCDM problem under consideration. The guideline we suggest presently is to ask some experts to provide these bounds necessary to the SPOTIS method, and then to make eventually a sensitivity analysis of the SPOTIS result with respect to the changes of the bounds for determining a margin of acceptable bound values to evaluate the robustness of the SPOTIS solution.

\section{E. Steps of SPOTIS method}

For convenience we summarize the main steps of SPOTIS method.
1) Step 1: Define the min and max bounds of classical (illdefined/incomplete) original MCDM problem in order to transform it into a well-defined MCDM problem ${ }^{12}$.

2) Step 2: Define the ideal solution point of MCDM depending of preference order of each criterion (larger is better, or smaller is better).

3) Step 3: For each alternative $A_{i}(i=1,2, \ldots, M)$, compute its normalized distance with respect to ideal solution for each criteria $C_{j}(j=1,2, \ldots, N)$ by either formulas (2) or (5).

4) Step 4: For each alternative $A_{i}(i=1,2, \ldots, M)$, compute its normalized averaged distance with respect to multi-criteria ideal solution by either formulas

$$
d\left(A_{i}, s^{\star}\right)=\sum_{j=1}^{N} w_{j} d_{i j}\left(A_{i}, s_{j}^{\star}\right)
$$

or equivalently

$$
\tilde{d}\left(A_{i}, S^{\star}\right)=\sum_{j=1}^{N} w_{j} \tilde{d}_{i j}\left(A_{i}, S_{j}^{\star}\right)
$$

5) Step 5: Sort alternatives in increasing order using $d\left(A_{i}, s^{\star}\right)$ (or equivalently $\tilde{d}\left(A_{i}, S^{\star}\right)$ ) values. The least value corresponds to the best MCDM solution $A^{\star}$, that is $A^{\star}=A_{i^{\star}}$, where $i_{\star}=\arg \min d\left(A_{i}, s^{\star}\right)$. The second least value corresponds to the second best MCDM solution, etc.

Once the MCDM is well-defined thanks to the specification of the bounds values of each criteria, the SPOTIS method does not suffer from rank reversal because the evaluation of each alternative is done independently of the others. Therefore, removing an alternative or including a new alternative in the new well-defined MCDM problem will not change the preference order of alternatives. The SPOTIS method must be adapted if the chosen ISP is a particular Expected Solution Point (ESP) that does not include maximum or minimum bounds of criteria. This is briefly presented in section $\mathrm{V}$.

\section{EXAMPLES FOR SPOTIS METHOD}

\section{A. Example 1 (continued)}

For this example, the ideal best solution is $S^{\star}=$ $\left(S_{1}^{\star}, S_{2}^{\star}, S_{3}^{\star}\right)=\left(S_{1}^{\max }, S_{2}^{\min }, S_{3}^{\max }\right)=(12,-6,5)$ because one considers that for criteria $C_{1}$ and $C_{3}$ the preference is larger score value is better, and for criterion $C_{2}$ the preference is smaller score value is better, and we have chosen the min and max bounds of criteria as $\left[S_{1}^{\min }, S_{1}^{\max }\right]=[-5,12]$, $\left[S_{2}^{\min }, S_{2}^{\max }\right]=[-6,10]$ and $\left[S_{3}^{\min }, S_{3}^{\max }\right]=[-8,5]$.

\footnotetext{
${ }^{12}$ The choice of the min and max bounds is left to the analyst and the system designer, and it highly depends on the MCDM problem they have to solve.
} 
The step 3 of SPOTIS yields the following normalized distances matrix

$$
\begin{aligned}
& \begin{array}{lll}
C_{1} & C_{2} & C_{3}
\end{array} \\
& \mathbf{d}_{i j}=\left[d_{i j}\left(A_{i}, s_{j}^{\star}\right)\right] \approx \begin{array}{c}
A_{1} \\
A_{2} \\
A_{3}
\end{array}\left[\begin{array}{lll}
0.0882 & 0.1812 & 0.2538 \\
0.2294 & 0.3750 & 0.1231 \\
A_{4} & 0.3937 & 0.2846 \\
0.5176 & 0.8313 & 0.7923
\end{array}\right]
\end{aligned}
$$

For instance, the value $d_{11}\left(A_{1}, s_{1}^{\star}\right) \approx 0.0882$ is obtained by the equivalent formulas (3) or (6), that is

$$
d_{11}\left(A_{1}, s_{1}^{\star}\right)=\frac{\left|S_{11}-S_{1}^{\star}\right|}{\left|S_{1}^{\max }-S_{1}^{\min }\right|}=\frac{|10.5-12|}{|12-(-5)|} \approx 0.0882
$$

The step 4 of SPOTIS yields the following normalized average distances $d\left(A_{i}, s^{\star}\right)=\sum_{j=1}^{N} w_{j} d_{i j}\left(A_{i}, s_{j}^{\star}\right)$

$$
\mathbf{d}=\left[d\left(A_{i}, s^{\star}\right)\right] \approx \begin{gathered}
A_{1} \\
A_{2} \\
A_{3} \\
A_{4}
\end{gathered}\left[\begin{array}{l}
0.1989 \\
0.37063 \\
0.7491
\end{array}\right]
$$

Sorting the distances vector $\mathbf{d}$ in ascending order we get

$$
d\left(A_{1}, s^{\star}\right)<d\left(A_{3}, s^{\star}\right)<d\left(A_{2}, s^{\star}\right)<d\left(A_{4}, s^{\star}\right)
$$

which means that $A_{1}$ is the closest alternative to ISP. The final preference order result of SPOTIS method for this example is therefore:

$$
A_{1} \succ A_{3} \succ A_{2} \succ A_{4}
$$

Suppose now that we take out one alternative, say $A_{2}$, of the MCDM problem for this example. Then, we have to consider now the following modified (reduced) score matrix

$$
\begin{aligned}
& \begin{array}{lll}
C_{1} & C_{2} & C_{3}
\end{array} \\
& \mathbf{S}_{\text {reduced }}=\begin{array}{c}
A_{1} \\
A_{3} \\
A_{4}
\end{array}\left[\begin{array}{ccc}
10.5 & -3.1 & 1.7 \\
8.1 & 0.3 & 1.3 \\
3.2 & 7.3 & -5.3
\end{array}\right]
\end{aligned}
$$

Applying SPOTIS steps 3 and 4 we gets the same normalized distances and average distances for the alternatives of the reduced MCDM problem, that is

$$
\begin{aligned}
& \begin{array}{ccc}
C_{1} & C_{2} & C_{3}
\end{array}
\end{aligned}
$$

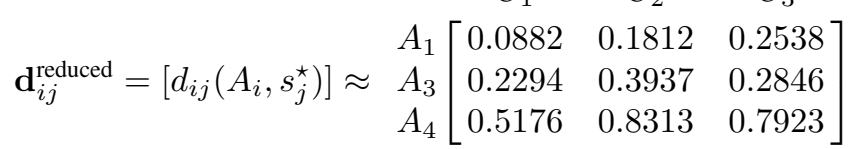

and

$$
\mathbf{d}^{\text {reduced }}=\left[d\left(A_{i}, s^{\star}\right)\right] \approx \begin{gathered}
A_{1} \\
A_{3} \\
A_{4}
\end{gathered}\left[\begin{array}{l}
0.1989 \\
0.74963
\end{array}\right]
$$

from which we have

$$
d\left(A_{1}, s^{\star}\right)<d\left(A_{3}, s^{\star}\right)<d\left(A_{4}, s^{\star}\right)
$$

and one deduces the final preference order

$$
A_{1} \succ A_{3} \succ A_{4} \text {. }
$$

which is naturally consistent with the previous result, i.e. there is no rank reversal.

Similarly, suppose we introduce a new alternative $A_{5}$ compatible with min and max bounds of criteria in the MCDM problem so that the modified (augmented) MCDM problem is characterized by the following (augmented) score matrix as follows

$$
\begin{aligned}
& C_{1} \quad C_{2} \quad C_{3}
\end{aligned}
$$

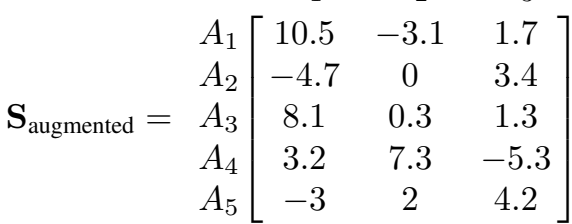

From SPOTIS steps 3 and 4 we get now

$$
\begin{aligned}
& C_{1} \quad C_{2} \quad C_{3} \\
& A_{1}\left[\begin{array}{lll}
0.0882 & 0.1812 & 0.2538
\end{array}\right]
\end{aligned}
$$



$$
\begin{aligned}
& \begin{array}{l|lll}
A_{4} & 0.5176 & 0.8313 & 0.7923 \\
A_{5} & 0.8824 & 0.5000 & 0.0615
\end{array}
\end{aligned}
$$

and

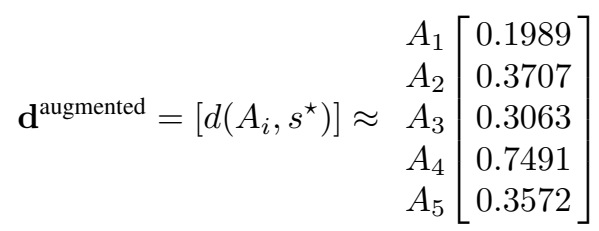

from which we have

$d\left(A_{1}, s^{\star}\right)<d\left(A_{3}, s^{\star}\right)<d\left(A_{5}, s^{\star}\right)<d\left(A_{2}, s^{\star}\right)<d\left(A_{4}, s^{\star}\right)$

and one deduces the final preference order

$$
A_{1} \succ A_{3} \succ A_{5} \succ A_{2} \succ A_{4} \text {. }
$$

which is also naturally consistent with the previous results of preference orderings, i.e. there is no rank reversal.

Suppose that the score values of $A_{5}$ meet exactly the ISP, that is $S_{5,1}=12, S_{5,2}=-6$ and $S_{5,3}=5$ then it is naturally expected that $A_{5}$ will be the most preferred alternative. It can be easily verified that this is exactly the solution that SPOTIS method provides because in this case

$$
\begin{aligned}
& C_{1} \quad C_{2} \quad C_{3} \\
& A_{1}\left[\begin{array}{lll}
0.0882 & 0.1812 & 0.2538
\end{array}\right] \\
& \begin{array}{l|lll}
A_{2} & 0.9824 & 0.3750 & 0.1231
\end{array}
\end{aligned}
$$

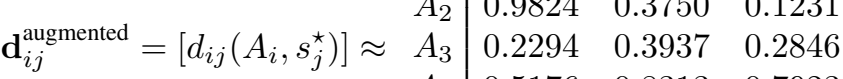

$$
\begin{aligned}
& A_{4} \quad 0.5176 \quad 0.8313 \quad 0.7923 \\
& A_{5}[0
\end{aligned}
$$

and the averaged normalized distances

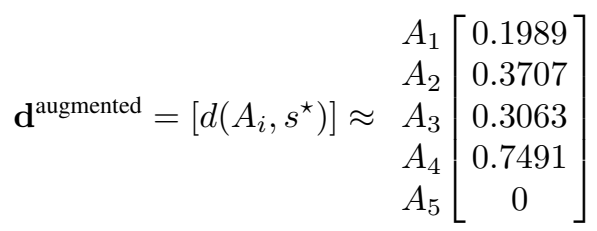


It can be also verified that if the score values of $A_{5}$ meet exactly the Worst Solution Point (WSP) when $S_{5,1}=-5$, $S_{5,2}=10$ and $S_{5,3}=-8$, then the alternative $A_{5}$ will become the least preferred solution provided by SPOTIS method which makes perfectly sense with what is naturally expected. Indeed, we will get in this case

$$
\left.\mathbf{d}^{\text {augmented }}=\left[d\left(A_{i}, s^{\star}\right)\right] \approx \begin{array}{c|c}
A_{1} & 0.1989 \\
A_{2} & 0.3707 \\
A_{3} & 0.3063 \\
A_{4} & 0.7491 \\
A_{5} & 1
\end{array}\right]
$$

\section{B. Example 2 (Car selection problem)}

Here we examine a more concrete example analyzed in [22] about car selection MCDM problem. We consider a set of four cars $\left\{A_{1}, A_{2}, A_{3}, A_{4}\right\}$ as follows:

- $A_{1}=$ TOYOTA YARIS 69 VVT-i Tendance;

- $A_{2}=$ SUZUKI SWIFT MY15 1.2 VVT So'City;

- $A_{3}=$ VOLKSWAGEN POLO 1.060 Confortline;

- $A_{4}=$ OPEL CORSA 1.4 Turbo 100 ch Start/Stop Edition

We consider the following five criteria for making the choice of the best car to buy:

- $C_{1}$ is the price (in $€$ );

- $C_{2}$ is fuel consumption (in $\mathrm{L} / \mathrm{km}$ );

- $C_{3}$ is the $\mathrm{CO} 2$ emission (in $\mathrm{g} / \mathrm{km}$ );

- $C_{4}$ is the fuel tank volume (in $\mathrm{L}$ );

- $C_{5}$ is the trunk volume (in $\mathrm{L}$ );

The score matrix $\mathbf{S}=\left[S_{i j}\right]$ is built from information extracted from car-makers technical characteristics available on the world wide web ${ }^{13}$. For the chosen cars, the corresponding score matrix is given by

$$
\mathbf{S}=\left[\begin{array}{ccccc}
15000 & 4.3 & 99 & 42 & 737 \\
15290 & 5.0 & 116 & 42 & 892 \\
15350 & 5.0 & 114 & 45 & 952 \\
15490 & 5.3 & 123 & 45 & 1120
\end{array}\right]
$$

We consider that the min and max bounds for each criterion are as follows

$$
\begin{aligned}
{\left[S_{1}^{\min }, S_{1}^{\max }\right] } & =[14000,16000] & \text { (in } €) \\
{\left[S_{2}^{\min }, S_{2}^{\max }\right] } & =[3,8] & (\text { in } \mathrm{L} / \mathrm{km}) \\
{\left[S_{3}^{\min }, S_{3}^{\max }\right] } & =[80,140] & (\text { in } \mathrm{g} / \mathrm{km}) \\
{\left[S_{4}^{\min }, S_{4}^{\max }\right] } & =[35,60] & (\text { in L) } \\
{\left[S_{5}^{\min }, S_{5}^{\max }\right] } & =[650,1300] & \text { (in L) }
\end{aligned}
$$

For criteria $C_{1}, C_{2}$ and $C_{3}$ the smaller is better. For criteria $C_{4}$ and $C_{5}$ the larger is better, so that the ISP is given by

$$
S^{\star}=(14000,3,80,60,1300)
$$

For simplicity, the importance $\operatorname{imp}\left(C_{j}\right)$ of each criteria $C_{j}$ takes a value in $\{1,2,3,4,5\}$, where 1 means the least important, and 5 means the most important. In this example we take $i m p\left(C_{1}\right)=5, i m p\left(C_{2}\right)=4, i m p\left(C_{3}\right)=4, i m p\left(C_{4}\right)=1$ and

\footnotetext{
${ }^{13}$ http://www.choisir-sa-voiture.com
}

$\operatorname{imp}\left(C_{5}\right)=3$ which means that the price of a car (criteria $C_{1}$ ) is the most important criteria for us, and the volume of fuel tank (criteria $C_{4}$ ) is the least important one. From these importance values and after normalization, we get the following vector of relative weights of criteria

$$
\begin{aligned}
\mathbf{w} & =\left[\begin{array}{lllll}
\frac{5}{17} & \frac{4}{17} & \frac{4}{17} & \frac{1}{17} & \frac{3}{17}
\end{array}\right] \\
& =\left[\begin{array}{lllll}
0.2941 & 0.2353 & 0.2353 & 0.0588 & 0.1765
\end{array}\right]
\end{aligned}
$$

Intuitively, based on the score matrix $\mathbf{S}$ and importances of criteria, the choice of car $A_{1}$ is anticipated to be the best choice because the three most important criteria meet clearly the highest values for the car $A_{1}$.

If we apply the SPOTIS method for this MCDM problem, we get

$$
\begin{aligned}
& \begin{array}{llllll}
C_{1} & C_{2} & C_{3} & C_{4} & C_{5}
\end{array}
\end{aligned}
$$

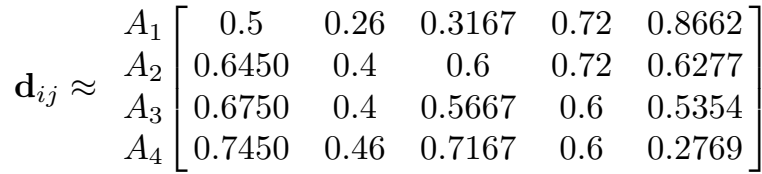

and the weighted average distances to ISP as follows

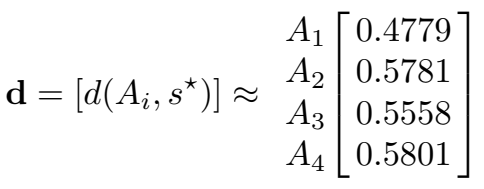

Hence the SPOTIS preference ordering is

$$
A_{1} \succ A_{3} \succ A_{2} \succ A_{4}
$$

which fits with what we expect because the car $A_{4}$ has the worst score for the most important criterion $C_{1}, C_{2}$ and $C_{3}$.

If we apply the classical TOPSIS approach [13], [14], one gets $A_{4} \succ A_{1} \succ A_{3} \succ A_{2}$, that is $A_{4}$ would be the best car to buy, whereas $A_{2}$ would be the worst one. TOPSIS result is not good and counter-intuitive because in this very simple and concrete example $A_{1}$ should have been selected as the best choice without ambiguity by any rational decision-maker.

With BF-TOPSIS methods (1, 2, 3 and 4) [22] we get the same satisfactory preference order $A_{1} \succ A_{3} \succ A_{2} \succ A_{4}$, which also coincides with AHP solution [12], and with the SAW (Simple Additive Weighting) method [8], [23] in this example. But contrary to aforementioned methods, SPOTIS approach is by construction free of rank reversal once the min and max bounds of criteria have been chosen by the MCDM system designer, and SPOTIS approach is very simple to apply which gives an advantage with respect to other MCDM methods.

It could be argued that the SPOTIS method is more difficult (or risky) to use because of the freedom left in the choice of min and max bounds of the criteria. We consider that this is not a very serious problem of SPOTIS method because in most of practical problems we have good insight of the (physical) bounds/limits of the criteria involved in the MCDM, 
and because a sensibility analysis of the choice of the bounds can always be conducted to see how SPOTIS method behave for the ill-defined MCDM problem if one doubts on the chosen bounds of the criteria. What is the most important is that SPOTIS is RRF once the original ill-defined MCDM problem has been transformed into a well-defined MCDM thanks to the choice of the criteria bounds.

\section{APPlying SPOTIS With A CHOSEN ESP}

In this section we show that SPOTIS method can also be applied (if one wants) using any chosen Expected Solution Point (ESP) rather than using the best Ideal Solution Point (ISP). The only condition is that each coordinate $S_{j}^{\star}$ of ESP must be between the bounds $\left[S_{j}^{\min }, S_{j}^{\max }\right]$ of each criteria $C_{j}$, $j=1,2, \ldots, N$ of the well-formulated MCDM problem. We illustrate the application of the SPOTIS method working with ESP in the previous MCDM car example for convenience.

Example 2 (continued): We consider the same MCDM car problem as in example 2, but we are now interested in the preference ordering of the four cars with respect to a chosen expected car that would satisfy our following five desiderata for each criteria

$$
S^{\star}=\left[\begin{array}{ll}
15300411550900
\end{array}\right]
$$

$S^{\star}$ corresponds to our chosen expected solution point.

We can verify that the coordinates of the chosen ESP are between the chosen bounds of each criteria because $S_{1}^{\star}=15300 \in\left[S_{1}^{\min }, S_{1}^{\max }\right]=[14000,16000], S_{2}^{\star}=4 \in$ $\left[S_{2}^{\min }, S_{2}^{\max }\right]=[3,8], S_{3}^{\star}=115 \in\left[S_{3}^{\min }, S_{3}^{\max }\right]=[80,140]$, $S_{4}^{\star}=50 \in\left[S_{4}^{\min }, S_{4}^{\max }\right]=[35,60]$, and $S_{5}^{\star}=900 \in$ $\left[S_{5}^{\min }, S_{5}^{\max }\right]=[650,1300]$. For this car selection problem we recall that the score matrix is given by

$$
\mathbf{S}=\left[\begin{array}{ccccc}
15000 & 4.3 & 99 & 42 & 737 \\
15290 & 5.0 & 116 & 42 & 892 \\
15350 & 5.0 & 114 & 45 & 952 \\
15490 & 5.3 & 123 & 45 & 1120
\end{array}\right]
$$

and the weights of criteria are

$$
\mathbf{w}=\left[\begin{array}{lllll}
0.2941 & 0.2353 & 0.2353 & 0.0588 & 0.1765
\end{array}\right]
$$

We can apply the formula (3) to compute the normalized distance of each alternative to the expected solution point for each criteria. One gets the following distance matrix $\mathbf{d}_{i j}=$ $\left[d_{i j}\left(A_{i}, s_{j}^{\star}\right)\right]$ whose numerical components are

$$
\begin{aligned}
& \begin{array}{llllll}
C_{1} & C_{2} & C_{3} & C_{4} & C_{5}
\end{array}
\end{aligned}
$$

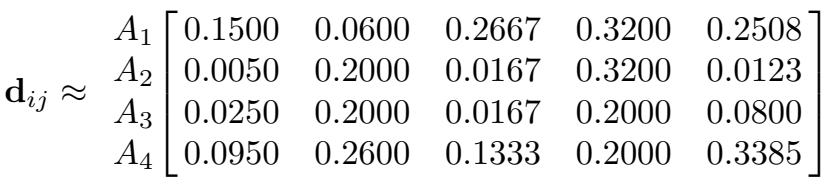

After obtaining their weighted average (step 4 of SPOTIS method), we get finally

$$
\left.\mathbf{d}=\left[d\left(A_{i}, s^{\star}\right)\right] \approx \begin{array}{c}
A_{1}\left[\begin{array}{l}
0.1841 \\
A_{2} \\
A_{3} \\
A_{4}
\end{array}\right] 0.0734 \\
0.1924
\end{array}\right]
$$

Therefore the SPOTIS preference ordering of cars based on this chosen ESP is

$$
A_{2} \succ A_{3} \succ A_{1} \succ A_{4}
$$

This result makes intuitively sense because the car $A_{2}$ (Suzuki car) is very close to ESP coordinate $S_{1}^{\star}$ for the most important criterion $C_{1}$, and it is also quite close to coordinate $S_{3}^{\star}$ for a second most important criteria. Note that the normalized average distance 0.0734 for the first best choice $A_{2}$ and the normalized average distance 0.0842 for the second best choice $A_{3}$ are actually very close so that in this particular problem, one could consider both choices $A_{2}$ and $A_{3}$ acceptable from practical standpoint. To make the decision more easier one could also introduce an (or several) additional criterion of course.

This example may appear too simple for readers familiarized with more complicated MCDM problems, but we think it is sufficiently interesting to show how our SPOTIS method works in a classical problem. Application of this method for risk analysis in mountains will be investigated in some of our future research works.

\section{CONCLUSIONS}

In this work, we have presented a new Stable Preference Ordering Towards Ideal Solution (SPOTIS) method to solve MCDM problem. The SPOTIS method is free of rank reversal because of introduction of min and max bounds of each criteria entering in the definition of the well-formulated MCDM problem. This method is very simple to apply and it works also with any expected solution point chosen in the bounds of criteria. We have shown how it works on a simple car selection problem comparatively to other classical methods. The adaptation of this new SPOTIS method for working with missing and imprecise data is under investigation.

\section{APPENDIX 1}

Proof of theorem 1:

To prove that $d(\mathbf{x}, \mathbf{y})$ is a true distance, we must prove the four properties

1) Positiveness: $\forall(\mathbf{x}, \mathbf{y}) \in E^{2}, d(\mathbf{x}, \mathbf{y}) \geq 0$

2) Symmetry: $\forall(\mathbf{x}, \mathbf{y}) \in E^{2}, d(\mathbf{x}, \mathbf{y})=d(\mathbf{y}, \mathbf{x})$

3) Separation: $\forall(\mathbf{x}, \mathbf{y}) \in E^{2}, d(\mathbf{x}, \mathbf{y})=0 \Leftrightarrow \mathbf{x}=\mathbf{y}$

4) Triangular inequality: $\forall(\mathbf{x}, \mathbf{y}, \mathbf{z}) \in E^{3}$,

$$
d(\mathbf{x}, \mathbf{z}) \leq d(\mathbf{x}, \mathbf{y})+d(\mathbf{y}, \mathbf{z})
$$

Because $d_{1}\left(x_{1}, y_{1}\right)$ is a true distance defined in $E_{1}$, one has $d_{1}\left(x_{1}, y_{1}\right) \geq 0$ for all $\left(x_{1}, y_{1}\right) \in E_{1} \times E_{1}$, and because $w_{1} \geq 0$, one has $w_{1} d_{1}\left(x_{1}, y_{1}\right) \geq 0$. Similarly $d_{2}\left(x_{2}, y_{2}\right)$ being a true distance in $E_{2}$ and $w_{2} \geq 0$, one has always $w_{2} d_{2}\left(x_{2}, y_{2}\right) \geq 0$ for all $\left(x_{2}, y_{2}\right) \in E_{2} \times E_{2}$. Hence the quantity $w_{1} d_{1}\left(x_{1}, y_{1}\right)+$ $w_{2} d_{2}\left(x_{2}, y_{2}\right) \geq 0$, which proves the positiveness of $d(\mathbf{x}, \mathbf{y})$.

Because symmetry holds for $d_{1}$ and $d_{2}$, one has $\forall(\mathbf{x}, \mathbf{y}) \in$ $E^{2} d(\mathbf{x}, \mathbf{y})=w_{1} d_{1}\left(x_{1}, y_{1}\right)+w_{2} d_{2}\left(x_{2}, y_{2}\right)=w_{1} d_{1}\left(y_{1}, x_{1}\right)+$ $w_{2} d_{2}\left(y_{2}, x_{2}\right)=d(\mathbf{y}, \mathbf{x})$ which proves the symmetry property of $d(\mathbf{x}, \mathbf{y})$. Because separation holds for $d_{1}$ and $d_{2}$, that is 
$d_{1}\left(x_{1}, x_{1}\right)=0$ and $d_{2}\left(x_{2}, x_{2}\right)=0$, one has $\forall(\mathbf{x}, \mathbf{x}) \in E^{2}$ the following equality $d(\mathbf{x}, \mathbf{x})=w_{1} d_{1}\left(x_{1}, x_{1}\right)+w_{2} d_{2}\left(x_{2}, x_{2}\right)=$ $w_{1} \cdot 0+w_{2} \cdot 0=0$, which proves the separation property of $d(\mathbf{x}, \mathbf{y})$. Let's verify also that the triangular inequality holds. Because $d_{1}$ and $d_{2}$ are true distances, they satisfy the triangular inequalities. That is, for all $\left(x_{1}, y_{1}, z_{1}\right) \in E_{1} \times E_{1} \times E_{1}$

$$
d_{1}\left(x_{1}, z_{1}\right) \leq d_{1}\left(x_{1}, y_{1}\right)+d_{1}\left(y_{1}, z_{1}\right)
$$

and for any multiplicative factor $w_{1} \geq 0$, one has also

$$
w_{1} d_{1}\left(x_{1}, z_{1}\right) \leq w_{1} d_{1}\left(x_{1}, y_{1}\right)+w_{1} d_{1}\left(y_{1}, z_{1}\right)
$$

Similarly, one has for any multiplicative factor $w_{2} \geq 0$

$$
w_{2} d_{2}\left(x_{2}, z_{2}\right) \leq w_{2} d_{2}\left(x_{2}, y_{2}\right)+w_{2} d_{2}\left(y_{2}, z_{2}\right)
$$

By adding the two positive (or null) left-hand sides, and the two positive (or null) right-hand sides of the previous inequalities, we get (after rearranging terms) the following inequality which is always valid

$$
\begin{gathered}
w_{1} d_{1}\left(x_{1}, z_{1}\right)+w_{2} d_{2}\left(x_{2}, z_{2}\right) \leq \\
w_{1} d_{1}\left(x_{1}, y_{1}\right)+w_{2} d_{2}\left(x_{2}, y_{2}\right)+w_{1} d_{1}\left(y_{1}, z_{1}\right)+w_{2} d_{2}\left(y_{2}, z_{2}\right)
\end{gathered}
$$

This valid inequality can be expressed equivalently as $d(\mathbf{x}, \mathbf{z}) \leq d(\mathbf{x}, \mathbf{y})+d(\mathbf{y}, \mathbf{z})$, which proves that $d(\mathbf{x}, \mathbf{y})$ satisfies the triangular inequality for all $(\mathbf{x}, \mathbf{y}, \mathbf{z}) \in E^{3}$. This completes the proof of the theorem.

By induction, this proof can be directly extended to the general case involving $n>2$ metric spaces, proving that for any $w_{i} \geq 0$ and using any distance $d_{i}$ chosen in $E_{i}, i=$ $1,2, \ldots, n$,

$$
d(\mathbf{x}, \mathbf{y})=\sum_{i=1}^{n} w_{i} d_{i}\left(x_{i}, y_{i}\right)
$$

is also a true distance.

\section{REFERENCES}

[1] V. Belton, T. Gear, "On a shortcoming of Saaty's method of analytic hierarchies," Omega, Vol. 11, No. 3, pp. 228-230, 1983.

[2] T.J. Stewart , "A critical survey on the status of multiple criteria decision making theory and practice," Omega International Journal of Management Science, Vol. 20, No.5-6, pp. 569-586, 1992.

[3] J. Barzilai, B. Golany, "AHP rank reversal, normalization and aggregation rules," Inform, Vol. 32, No. 2, pp. 57-63, 1994.

[4] E. Triantaphyllou, Multi-criteria decision making methods: a comparative study, Kluwer Academic Publishers, Dordrecht, 2000.

[5] D. Pavlicic, "Normalization affects the results of MADM methods," Yugoslav J. of Operations Research, Vol. 11, No. 2, pp. 251-265, 2001.

[6] E.K. Zavadskas, A. Zakarevicius, J. Antucheviciene, "Evaluation of ranking accuracy in multi-criteria decisions," Informatica 17, pp. 601618, 2006.

[7] X. Wang, E. Triantaphyllou, "Ranking irregularities when evaluating alternatives by using some ELECTRE methods," Omega, Vol. 36, No. 1, pp. 45-63, 2008.

[8] Y.-M. Wang, Y. Luo, "On rank reversal in decision analysis," Mathematical and Computer Modelling, Vol. 49, pp. 1221-1229, 2009.

[9] H. Maleki, S. Zahir, "A comprehensive literature review of the rank reversal phenomenon in the analytic hierarchy process," J. of MultiCriteria Decision Analysis, Vol. 20, pp. 141-155, 2013.

[10] Y.B. Shin, S. Lee, S.G. Chun, D. Chung, "A critical review of popular multi-criteria decision making methodologies," Issues in Information Systems, Vol. 14, No. 1, pp. 358-365, 2013.
[11] R. Felinto de Farias Aires, L. Ferreira, "The rank reversal problem in multi-criteria decision making: a literature review," Pesquisa Operacional, 38(2), pp. 331-362, 2018

[12] T. Saaty, The analytic hierarchy process, McGraw-Hill, 1980.

[13] C.L. Hwang, K. Yoon, "Multiple attribute decision making," Lecture Notes in Economics and Math. Syst. 186, Springer-Verlag, Berlin, 1981.

[14] Y.J. Lai, T.Y. Liu, C.L. Hwang, C.L., “TOPSIS for MODM,” European Journal of Operational Research, Vol. 76, No. 3, pp. 486-500, 1994.

[15] H. Yin, J. Lan, X.-R.Li, "Measures for ranking estimation performance based on single or multiple performance metrics," Proc. of Fusion 2013 , Istanbul, Turkey, July 9-12, 2013.

[16] A. Piegat, W. Sałabun, "Identification of a multicriteria decision-making model using the characteristic objects method," Applied Computational Intelligence and Soft Computing, Volume 2014, Article ID 536492, 14 pages.

[17] W. Sałabun, "The characteristic objects method: a new distance-based approach to multicriteria decision-making problems," J. Multi-Crit. Decis. Anal., 2014.

[18] W. Sałabun et al., "Handling data uncertainty in decision making with COMET," Proc. of 2018 IEEE Symposium Series on Computational Intelligence (SSCI), Bangalore, India, Nov. 2018.

[19] P.L. Kunsch, "A statistical approach to complex multi-criteria decisions," Computational Intelligence in Complex Decision Systems (Da Ruan Editor), Atlantis Press, Chap. 6, pp. 147-182, 2010

[20] F. Kong, "Rank reversal and rank Preservation in TOPSIS," Advanced Materials Research, Vols. 204-210, pp. 36-41, 2011.

[21] H. Anton, Elementary linear algebra, John Wiley \& Sons (7th Ed.), New York, 1994.

[22] J. Dezert, D. Han, H. Yin, "A new belief function based approach for multi-criteria decision-making support," Proc. of Fusion 2016, Heidelberg, Germany, July 2016.

[23] C.L. Hwang, K. Yoon, Multiple attribute decision making methods and applications, Springer-Verlag, New York, 1981. 